\title{
Homogenous Cosmos Originated from Unique Genesis Precondition Initialization of PNT dynamics
}

\author{
Guo-sheng Yang \\ PingYuan vallige, Zhenzhu town, Wugang city, Hunan province, China \\ Marktin-yang@163.com
}

Keywords: Homogenous cosmos originated from unique genesis postulate, Space field, time dynamic speed, positive \& negative transmutation (IPNT), absolute spacetime momentum.

\begin{abstract}
Remarkable progress of modern particle physics has thrown more and more lights onto factuality that any discretionary particles in cosmos are mutually convertible, and living progress of it even makes the factuality orient perfect induction day by day, which apparently implies that cosmos is throughout homogenous originated from unique genesis. And on the basis of integrative precondition of "homogenous cosmos originated from unique genesis + spacetime \& matter coequally belong to category of cosmos", it logically arrives at "logic extension 1 of Homogenous cosmos originated from unique genesis postulate" _ spacetime and matter are coequally physical. On the basis of integrative precondition of "homogenous cosmos originated from unique genesis + spacetime \& matter fully embodying cosmos", it logically arrives at "logic extension 2 of Homogenous cosmos originated from unique genesis postulate” __ spacetime and matter are homologic filiation. And subsequently, it introduces "connotation objectification of the two logic extensions of Homogenous cosmos originated from unique genesis postulate" to accomplish homology objectification of entire cosmos so as to highlight intrinsic homologic attribute of spacetime and matter, homologic filiation between spacetime and matter, even essence of matter, which are of course favorable rational groundwork for further logic extension towards qualitative redefinition and mathematical formulation of existence \& motion of matter in homogenous cosmos.
\end{abstract}

\section{Introduction of Homogenous cosmos originated from unique genesis postulate}

\subsection{Newly highlighted factuality of "any discretionary two particles in cosmos are mutually}

convertible" causes naissance of idea of homogenous cosmos originated from unique genesis

In the recent 100 years, as remarkable progress of modern particles physics, we've found more and more colorful particles in cosmos, we see any discretionary 2 particles selected from particle/object aggregation of $\left\{A_{1} 、 A_{2} 、 A_{3} 、 \ldots A_{n}(n \in N)\right\}$ are mutually convertible. (Which means any discretionary $C_{n}{ }^{2}$ option of the particle aggregation is mutually convertible). The cause is that the discretionary 2 particles/objects selected from the particle/object aggregation always possess the common compositive components in certain configurable hiberarchy.

Such factuality can lead us to approach such deduction, if the magnitude of $n(n \in N)$ is big enough so that the object aggregation of $\left\{A_{1} 、 A_{2} 、 A_{3} 、 \ldots A_{n}(n \in N)\right\}$ can represent the entire cosmic contents, and any discretionary 2 objects selected from the object aggregation are still mutually convertible, then the entire cosmic contents must possess the common extremity component in terminal configurable hiberarchy, — _ the cosmos is throughout homogenous made up of the common extremity component in terminal configurable hiberarchy.

And at the same time, since the entire cosmic contents possess the so-called "common extremity component" in their common terminal configurable hiberarchy, the so-called "common extremity component" in cosmos must be unique, because there is no possibility for two or more different components to take the role of "common extremity component". This of course implies the entire cosmic contents originated from the "common and unique extremity hylic progenitor /component", any cosmic content is either he unique extremity hylic progenitor (or genic origin) itself or 
homologous compound radically originated from the common unique extremity hylic progenitor (or genic origin). It of course implies the entire cosmos is not only throughout homogenous but also absolute homology aggregation systematically originated from unique genesis.

However, we have to admit the conclusion any discretionary 2 particles/objects in cosmos are mutually convertible is still our experiential summary based on millions of but still inadequate positive facts that history has already offered. The rational situation is rather similar as a mathematical induction. In human history, we have procured substantial facts which support, when $n=1,2,3, \ldots$, $\mathrm{k}$, all situations satisfy conclusion that any discretionary 2 objects in the particle/object aggregation $\left\{A_{1}, A_{2}, A_{3}, \ldots A_{k}(k \in N)\right\}$ are mutually convertible, and as history continuously extending, we found more and more new particles in cosmos, the magnitude of $\mathrm{k}$ is proportionally increasing, all situations still satisfy the conclusion. Whereas, once we suppose the magnitude of $n$ is big enough so that the particle/object aggregation $\left\{A_{1}, A_{2}, A_{3} \ldots A_{n}(n \in N)\right\}$ can be exactly equal to cosmic contents, whether all situations still satisfy the conclusion?

Confronting challenge of the very issue, we have to admit that the conclusion "any discretionary two particles in cosmos are mutually convertible" is still a conclusion of unperfect induction. Although it has been remarkably highlighted by K experimental facts which have been perceived in the recent one century, it is still unperfect for there are still $n-k(n \in N, n>k)$ facts out of demonstration, —a conclusion of unperfect induction.

Whereas, is it possible for cosmos to be really homogenous originated from unique genesis through and through? Since we still lack of credible backup for our positive conclusion, confronting challenge of the very issue, should we flinch or not?

No! No No No, leastways, as temporal practical limitation of human, the conclusion any discretionary two particles in cosmos are mutually convertible is still out of perfect induction does not directly indicate potential flimflam that there must be some certain two potential particles in cosmos not mutually convertible undoubtedly. At any rate, we have to admit it's impossible for human practice to be always consummate \& satisfying for theoretical necessity of scientific exploration on the spot, temporal limitation of human practice for further exploration is inevitable. And on the other hand, something even all-important is that it's still necessary for us to consider whether the conclusion of factuality any discretionary two particles in cosmos are mutually convertible is still in living orientation towards perfect induction as proceeding of human practice, and something even all-important is that it's still necessary for us to consider whether introduction of the presumable conclusion really significant for further theoretical exploration of science. Otherwise, we are substantively recreant in scientific pursuit.

Virtually, no matter on stand of active practice or rational necessity of scientific exploration, we should not flinch for the living conclusion still out of perfect induction. After all, the positive conclusion of the very issue is really all-important for us to look into the grand cosmological authenticity. At any rate, all the particles we perceived in the recent one century are mutually convertible, none is exceptional, and as time going itself, we perceived more and more positive facts supporting the conclusion of the factuality, this of course indicates the conclusion of the very factuality in living orientation towards perfect induction.

Confronting challenge of the very issue whether cosmos is throughout homogenous originated from unique genesis or not, if we say "Yes", we of course have substantial positive evidence; inversely, if we say "No", nothing seems favorable for rational support.

If cosmos is really homogenous originated from unique genesis through and through, then, the thoroughpaced configuration of cosmos will be remarkably illuminated, _ _ intrinsic attribute and inherent correlation of spacetime \& matter, consequential existence \& motion of matter in spacetime due to the proper intrinsic attribute and inherent correlation of spacetime \& matter will immediately be self-evident followed a rational line of cosmic demiurgic evolution of sequential homologic multiplication starting at the so-called unique extremity progenitor component to cosmic status quo. At any rate, essence of matter generally come into being during the course of their original generation \& formation and is inherently kept with matter existing as idiographic objects under the 
proper generative conditions. The magic significance of exploration endeavor towards the very issue is rather similar as that we are looking for a very long string in terrible darkness, as our eyeshot is too dark, we are almost confused unknowing where to go and what to do, However, as an innovative and enterprising attempt (such as pioneering introduction of Homogenous cosmos originated from unique genesis postulate), one terminal of the long string in terrible darkness has suddenly been caught hold of by us, the rest job is but to pull the string and approach all the necessary with the string in hands, and terrible darkness virtually means nothing to us ever since.

Considering the logic significance of the very issue for further scientific exploration, in order not to intermit the positive scientific pursuit of intrinsic attribute \& inherent correlation of spacetime \& matter even universal existence \& motion of matter in spacetime along the very rational clew, it seems wise for us to ascertain the potential conclusion with living orientation towards perfect induction as presumable precondition so as to release pioneering endeavor of scientific pursuit from our temporal limitation. We consequently introduce a presumable precondition of "Homogenous cosmos originated from unique genesis postulate" herein. At any rate, on exploration journey of science, preferential introduction of intelligent postulate is usually ready to release pioneering endeavor from temporal practice limitation towards expedient scientific pursuit, whose practical significance mostly indwells, as intelligent and timely introduction of the proper postulate, how much of our goal rational eyeshot has been swiftly illuminated and widely open thereby.

\subsection{Homogenous cosmos originated from unique genesis postulate}

It is assumed that cosmos is throughout homogenous originated from unique genesis. In detailed words, entire cosmic contents are all congenitally coherent homologies consisting in an absolute homogeneity system which originally originated from unique genesis along cosmic demiurgic evolution of sequential homologic multiplication starting at so-called "unique genesis" to cosmic status quo through and through. Cosmic contents are either the so-called "unique genesis (cosmic extremity genic component/cosmic genic origin)" itself or idiographic homologous compounds with distinctive homologic configurations in cosmic homogeneity system.

The introduction of Homogenous cosmos originated from unique genesis postulate radically roots in conclusion of potential factuality that any discretionary two particles in cosmos are mutually convertible. Upon our strict sense, the reason that Homogenous cosmos originated from unique genesis postulate can only be a "postulate" is but because conclusion that any discretionary two particles in cosmos are mutually convertible is still experiential conclusion with living orientation towards perfect induction but still out of perfect induction. Whereas, the introduction of Homogenous cosmos originated from unique genesis postulate is substantively like a powerful thunderbolt in darkness of our rational eyeshot of cosmology, which illuminates thorough configuration of cosmos in a sudden that cosmos is throughout homogenous originated from unique genesis, so that, for the rational highlight, we can expediently look into homologic attribute of spacetime and matter, homologic correlation between spacetime and matter and existence \& motion of matter in homogenous cosmos following a rational line of cosmic demiurgic evolution of sequentiaal homologic multiplication towards cosmic status quo.

Of course, Homogenous cosmos originated from unique genesis postulate is logic origin and general precondition of the whole subject, it's also the basal logic fulcrum of the subject and rational reference that traditionary sciences (especially modern particle physics) have gradually highlighted for Homogenous Cosmos Originated from Unique Genesis by stochastical recognizant cumulation of serial microcosmic configuration of matter in the recent 100 years. The kernel of the basal logic fulcrum is that any discretionary two particles in cosmos are mutually convertible, in a straightforward word, Homogenous Cosmos Originated from Unique Genesis is but cosmos redefinition radically extended from newly highlighted factuality of any discretionary two particles in cosmos are mutually convertible. Inversely, if somebody are able to find such two objects (or such two particles) in cosmos, which can be fully proved there is absolutely no way for them to be converted each other, then, he either has radically negated the factuality cosmos is throughout 
homogenous originated from unique genesis or he has practically found tangible cosmos II already.

\subsection{Logic extension of "Cosmos is throughout homogenous originated from unique genesis + spacetime matter fully embodying cosmos"}

What a homogenous cosmos is it on earth?

If we only think of the Homogenous cosmos originated from unique genesis postulate only, we can hardly perceive something hypostatic aboout cosmos. after all, Homogenous cosmos originated from unique genesis postulate is but a nonrepresentational generalization about cosmic profile, whose ideographic efficacy is substantively like something offered by a couple cosmic mould cavities. Although a couple cosmic mould cavities really offers profile of cosmos, it's empty, it is not expedient for us to perceive the realistic profile of hypostatic cosmos. Since you insist that cosmos is homogenous originated from unique genesis through and through and the entire cosmos is completely an integrative cosmic homogeneity system, you should show us what are the hypostatic homologic components in cosmos which are congenitally correlated into coherent homologic integration as vivid cosmic homogeneity system all wool and a yard wide.

Towards the necessity of theoretical pursuit, we substitute the solid intuitional factuality that cosmos consists of spacetime and matter, none coordinate component else into Homogenous cosmos originated from unique genesis postulate so as to hypostatize the realistic homologic profile of cosmos with hypostatic cosmic endosome, so that we can expediently look into intrinsic homologic attribute of spacetime and matter, inherent homologic filiation between spacetime and matter and consequential existence $\&$ motion of matter in spacetime at the volley.

Upon our solid intuition, spacetime and matter are of course hypostatic contents of cosmos out of question, since we confirm cosmos is throughout homogenous originated from unique genesis, all cosmic contents must be homologies in cosmic homogeneity system with either direct pedigree correlation or indirect pedigree correlation . This of course means, as long as we have no tangible evidence to exclude spacetime and matter out of category of cosmos, both spacetime and matter must coequally belong to category of cosmic homogeneity system as homologic coordinators.

Thus, on the basis of integrative precondition of "homogenous cosmos originated from unique genesis + spacetime \& matter coequally belonging to category of cosmos", we can arrive at deduction as below:

Logic extension 1: Since cosmos is throughout homogenous originated from unique genesis, as undeniable components of cosmos, both "spacetime" and "matter" must be homologic coordinators coequally originated from the common cosmic extremity genesis (/cosmic genic origin) along cosmic original demiurgic evolution of sequential homologic multiplication,. And of course, as coordinative homology of matter, "spacetime" must be coequally physical as matter in cosmos, unless we have tangible evidence to exclude spacetime out of category of cosmos.

Moreover, upon our solid intuition, besides spacetime and matter, there seems nothing else coordinative in cosmos. However, is it really true that cosmos completely consists of spacetime and matter, none else?

In the face of challenge of the very issue, it seems, upon our solid intuition, we should be confident to draw our sensible conclusion that cosmos straightforwardly consists of spacetime and matter. Leastways, besides spacetime and matter, nothing else we've found in cosmos indeed, it even seems impossible for us to find a third coordinative component in cosmos. However, we are substantively difficult to prove the sensible conclusion fully upon our strict sense. If we reluctantly introduce the sensible conclusion as "truth" for further logic extension, we are substantively flimsy and bashful for we've bet too much of our intuition, which apparently seems rational perplexity as mute engluts lotus but unable to speak out his live taste.

In order to release pioneering scientific pursuit from pestilent rational perplexity for historical limitation in practice for the moment, we introduce an auxiliary postulate-spacetime matter fully embodying cosmos postulate, which postulates that cosmos is object fully and exactly embodied by hypostatic coexistence of coordinative spacetime and matter, nothing else. 
Apparently, in terms of theoretical function, "spacetime matter fully embodying cosmos postulate" and "Homogenous cosmos originated from unique genesis postulate" coequally outline profile of cosmos besides disparity of significance. If we think the ideographic significance of cosmic profile of Homogenous cosmos originated from unique genesis postulate a couple of mould cavities of cosmos, then, the ideographic significance of cosmic profile of spacetime matter fully embodying cosmos postulate is similar as an accessorial slide of the couple of mould cavities of Homogenous cosmos originated from unique genesis postulate. And just because of the necessity, we introduce spacetime matter fully embodying cosmos postulate as a coordinative auxiliary postulate of Homogenous cosmos originated from unique genesis.

Thus, on the basisi of integrative precondition of "Homogenous cosmos originated from unique genesis + spacetime matter fully embodying cosmos”, we can arrive at further logic extension as below:

Logic extension 2: If cosmos is object fully embodied by hypostatic coexistence of spacetime and matter, nothing else, along original cosmic demiurgic evolution of sequential homologic multiplication starting at the so-called common cosmic extremity genesis (/cosmic genic origin) to cosmic status quo, "spacetime” and "matter" must be homologic filiation.

This is just the primary two logic extensions of Homogenous cosmos originated from unique genesis postulate once we objectify spacetime and matter into Homogenous cosmos originated from unique genesis postulate. - on the basis of integrative precondition of "Homogenous cosmos originated from unique genesis + spacetime \& matter coequally belonging to cosmos", it educes "logic extension 1 of Homogenous cosmos originated from unique genesis postulate" spacetime and matter are coequally physical as coordinative homologies; on the basis of integrative precondition of "Homogenous cosmos originated from unique genesis + spacetime matter fully embodying cosmos", it educes "logic extension 2 of Homogenous cosmos originated from unique genesis postulate” — spacetime and matter are homologic filiation.

2 Connotation objectification of two logic extensions of Homogenous cosmos originated from unique genesis postulate, Coherent coexistence of "spacetime" \& "matter" as lineal homologic coordinators fully embodies cosmic homogeneity system.

2.1 Connotation objectification of logic extension 1 of Homogenous cosmos originated from unique genesis postulate, _ Homology hypostatization of spacetime as coordinator of matter in cosmic homogeneity system

\subsubsection{Objectification of homologic spacetime in cosmic homogeneity system}

(1) Spacetime genic field hypothesis

In accordance with logic extension 1 of Homogenous cosmos originated from unique genesis postulate, since it has been confirmed as sensible postulate that cosmos is throughout homogenous originated from unique genesis, as hypostatic components of cosmos, "spacetime" and "matter" must be coordinative homologies originated from the common cosmic extremity genesis along cosmic original demiurgic evolution of sequential homologic multiplication, and "spacetime" must be coequally physical as matter in cosmos, unless we have tangible evidence to exclude spacetime out of category of cosmos. Here, for the necessity and expedience of further analysis, we hypostatize the "hylic essence" of homologic spacetime into substantial aggregation of compositive element of homologous genic unit series coexisting as field, (and as the hylic existence of homologic spacetime consisting of the proper compositive genic units is very similar as field, we call spacetime "space field" too.) and we just call it "spacetime genic field hypothesis", which can be described as below;

It is hypothesized that space field consists of $n+1$ sorts of elementary genic components/units essentially originated from cosmic extremity genic origin along cosmic original demiurgic evolution of sequential homologic multiplication. We just call these elementary genic components /units in space field "field genic units", and separately symbolize them as $i_{0}, i_{1}, i_{2}, \ldots i_{n}(n \in N)$. And at the same time, we denote the relativistic configuration ratio of each genic unit in space field as a, 
$\mathrm{b}, \mathrm{c}, \ldots, \mathrm{x}$, so that we can primarily denote the integrative component configuration of field genic units in space field as $a * \mathrm{i}_{0}, \mathrm{~b} * \mathrm{i}_{1}, \mathrm{c} * \mathrm{i}_{2}, \ldots, \mathrm{x} * \mathrm{i}_{\mathrm{n}} .(\mathrm{n} \in \mathrm{N})$

(2): Phantasmal field genic unit hypothesis

Upon our common experience and solid intuition, any matter innately exists in dynamic and variable state, but never exists in stationary state; it even seems there is none reasonable ways for any matter to exist in ideal stationary and invariable state. According as the homologic essence of hylic spacetime in cosmic homogeneity system, it seems necessary and wise for us to extensionally complement the dynamic essentiality of hylic spacetime so as to consummate the physical existence of material space field. So we introduce Phantasmal field genic unit hypothesis to complement the dynamic essence of hylic spacetime as below:

It is hypothesized that all field genic units in space field exist in state of everlasting mutual transmutation and transition in transnormal speed, so that it's completely impossible for us to ascertain and perceive any definite presence of any separate field genic unit in space field, but only possible for us to perceive the holistic effect of their dynamic coexistence.

According as logic extension 1 of Homogenous cosmos originated from unique genesis postulate, homology hypostatization of spacetime in cosmic homogeneity system as coordinative homology of matter not only introduced "spacetime genic field hypothesis" to hypostatize the physical esse of hylic spacetime but also introduced "phantasmal field genic unit hypothesis" to extensionally complement the dynamic essence of hylic spacetime so as to consummate physical existence of homologic spacetime in cosmic homogeneity system.

And as introduction of innovative idea of homogenous cosmos originated from unique genesis, objectification of logic extension 1 of Homogenous cosmos originated from unique genesis postulate has virtually hypostatized spacetime as coordinative homology of matter in cosmic homogeneity system. In order to differentiate the hylic coordinative existence of homologic spacetime and matter in cosmic homogeneity system, we just call the material components of homologic spacetime "negative matter" and call the material components of matter we usually refer to "positive matter".

\subsection{2 "Time” and "Time Dynamic Speed”}

We now come to define "time" and "time dynamic speed" in accordance with the two hypotheses above.

According to space field genic hypothesis and Phantasmal field genic unit hypothesis above, it is very easy for us to know that the most hypostatic physical endosome of space field are but the hylic esse embodied by genic component configuration of certain homologic field genic unit series originated from the "cosmic common extremity genic origin" and the dynamic essence of transnormal dynamic transmutation \& transition among all field genic units.

So called "time" is just dynamic esse of hylic space field, —-the dynamic integration of mutual transmutation \& transition among all field genic units in space field. Time rate directly depends on rapidness and intensity of dynamic transmutation \& transition among all field genic units in space field. In different space fields, as difference of space field strength, the intensity and rapidness of dynamic transmutation \& transition among field genic units is proportionally different. Generally, the bigger the magnitude of space field strength is, the more intense and rapid the covariant transmutation \& transition among field genic units is, and the faster time goes therefore.

As integrated activity aggregation of dynamic transmutation and transition of field genic units in space field, time itself is virtually a special speed, —- the integrative rate of dynamic mutual transmutation and transition of all field genic units in space field. For convenience, we just call the integrative rate of dynamic mutual transmutation and transition of all field genic units in space field "time dynamic speed", and symbolize it with general character "Y". Time dynamic speed is just the symbolic logic style of the integrative intensity and rapidness of dynamic transmutation and transition of field genic units in space field. It adopts proper numerical value to denote the dynamic transmutation intensity and rapidness of field genic units according to physical significance \& necessity, which is a little similar as we adopt centigrade value to denote the intensity degree of 
thermo motion of molecules.

Then, how should we define "time dynamic speed" exactly?

It's well known, when we are ready to theoretically scale thermal degree of any objects, we propaedeutically adopt precondition definition of boiling water under standard atmosphere as $100^{\circ} \mathrm{C}$ as thermal quantification standard to scale and quantify thermal intensity of any objects. Whereas, once we come to scale the rate of time, (Just to define or quantify the rate of integrative dynamic intensity or rapidness of transmutation \& transition of field genic units in space field mathematically) the similar way is apparently not ideal.

let's suppose, if our study object just limits in one sole object, for instance, it's just the water, under standard atmosphere press and $20^{\circ} \mathrm{C}$, we define the cumulative length of linear thermal motion route of any water molecule in free water in one standard time unit "water thermal speed", (Note: here "free water" means water molecule never collides on any impurity molecules during the course of thermal motion.) Then, compared with centigrade value, water thermal speed not only has remarkable superiority of accuracy to represent the thermal degree, but also has remarkable superiority to incarnate the thermal hypostasis of molecule motion integration. Time dynamic speed can be defined by the similar way.

Thus, for definition expedience of time dynamic speed, it's necessary for us to introduce two related special items in advance.

(1). "Phoney motion"

Any water molecule is perceivable and relatively steady material object which we can track and ascertain at any moment; as a result, there must be tangible motion route during the course of thermal motion of molecule. Whereas, normal existence of any field genic unit is absolutely inconstant, so that it is completely impossible for us to ascertain and perceive their definite presence as explicit behavioral esse in time current, as a result, when they are coexisting and mutually transmuting in their innate state, there is none tangible motion route or orbit of any field genic unit. However, the transmutation and transition among field genic units can substantively lead to transposition among different field genic units. For instance, there are field genic units A and B in certain space field, when field genic units $A$ and $B$ transmute each other, genic unit A turns into genic unit B, and almost at the same time, genic unit B turns into genic unit $\mathrm{A}$, thus, the consequence of the transmutation between field genic unit A and field genic unit B is just equivalent to transposition between genic unit A and field genic unit B. We just call the very transposition caused by mutual transmutation among field genic units in the space field "phoney motion".

As long as the sorts of field genic units in space field are definite, as long as any two field genic units in the space field can convertibly transmute each other, the dynamic transmutation and transition among field genic units must lead to "phoney motion". And as the transposition among field genic units has no definite direction, but equivalent to any direction in ratio, the "phoney motion" has no direction, which of course indicates that the word "phoney" of phoney motion has double meanings, firstly, it indicates their actualization of transposition depends on mutual conversion, field genic units never transfer mechanically as independent esse, but as mutual conversion makes their behavioral effect equivalent to transference; secondly, the transposition never goes along certain direction. It's substantively a "phoney motion".

(2). "Standard time unit"

When we are ready to measure or quantify geometrical magnitude of any object, it's always necessary for us to choose a proper standard measure unit in prior. For instance, we choose "meter" as the standard length unit to measure discretionary length of any object or distance between any two points. But now, let's suppose the material of the ruler is rather sensitive at thermic intumescences, so that once we measure with this ruler under different ambient conditions of temperature, just because of discrepancy of thermic intumescences of the ruler in different temperature conditions, even though, the measure results we get might be just the same numeric magnitude, the practical length of objects we measured may be remarkably different. When we come to consider the measurement of time rate, as the disparity among different space fields, similar domino offect is even remarkable. 
As distinction of space field strength, the rate of time in different space field can never be the same. As time rate determines comprehensive motion rate of the matter existing in the proper space field including the rate of any clocks (It's easy to be comprehended according to definition of absolute spacetime momentum $\mathrm{mV}=\mathrm{d}(\mathrm{M} \leftarrow \mathrm{m}) \mathrm{Y}$ of any object in spacetime and the relevant PNT dynamics equations, such as acceleration formula $\mathrm{a}(\mathrm{t})=\mathrm{Y}(\mathrm{t})^{*} \mathrm{~K}^{\prime}(\mathrm{t})$, velocity formula $\mathrm{V}=\mathrm{V}_{0}+\Delta \mathrm{V}=\mathrm{V}_{0}+\int \mathrm{a}(\mathrm{t}) \mathrm{dt}$ etc), this of course indicates even just the same clock existing in different space field, as hypostatic distinction of space field strength, the connatural motion speed of the clock is impossible to be the same.

Let's suppose there are two aircrafts, they are just the same and each is fixed with a clock exactly the same too. And let's suppose just at the same moment the both aircrafts start at space field A and separately fly into the space field B and space field $C$ at the same initial speed. Standing in the space field A, we observe the both aircrafts, we find the both aircrafts separately fly into space field B and space field $C$ at the same moment and at the same initial speed, then we begin to record time in the space field A. 11 seconds later, we come to observe the both clocks on the two aircrafts, the results may be that the clock in space field $\mathrm{B}$ has gone 10 seconds, and the clock in the space field $\mathrm{C}$ has gone 12 seconds. And synchronously, standing in space field A, we come to measure the distance that the both aircrafts separately flown through in the "same interval", the results may be that the aircraft in the space field B has flown through 100 kilometers, and the aircraft in the space field C has flown through 120 kilometers. Although considered by the observer in space field A, the speed of the aircraft in space field $\mathrm{C}$ is 1.2 times of the speed of the aircraft in space field $\mathrm{B}$, if the cosmonauts can communicate with each other, then the flying speed of their own aircraft they mutually communicated themselves is just the same. (Just as 100 kilometers/10 seconds $=120$ kilometers/12 seconds). Even the both aircrafts transpose the space fields they fly in, as the change rate of aircraft flying speed and the change rate of the clock on the aircraft are exactly synchronous and equivalent, the cosmonaut in the aircraft has no way to find out the change of the flying speed of his aircraft, he still think his aircraft keeping in initial motion state constantly.

Just because of the very synchronous and equivalent domino offect time works on universal motion rate of matter in spacetime, when we come to measure the cosmic time in different space fields, it seems necessary for us to define a proper "standard time unit" for coherent quantification of time, but not to behave as ignorantly believe in the measurement according to the ruler itself regardless the thermic intumescences in different temperature conditions.

Then, what a "standard time unit" we should choose according to practical feasibility and necessity?

In accordance with practical feasibility and necessity, we confirm that under presumable precondition that the space field status of which the earth exists in is constant; the interval of 1 second on the earth is just one standard time unit. Apparently, as introduction of the "presumable precondition", we can nor ensure the so-called "standard time unit" is absolute constant without question, it but seems none better accessible for us to pursue.

Now, we come to define time dynamic speed. So called "time dynamic speed" is just mathematical quantification of phoney displacement cumulation of field genic units materialized by their mutual dynamic transmutation \& transition in one standard time unit. The magnitude of time dynamic speed not only directly indicates the time rate, but also implicates the integrative rate/capability of universal existence \& motion of matter in cosmos.

If we still adopt "meter" as the unit of phoney displacement and adopt "second" as the time unit, then, the unit of time dynamic speed is "meter/second" too. However, as the genic phoney displacement has no definite direction, time dynamic speed is a pure scalar quantity, it but quantitatively scales dynamic intensity \& rapidness of phantasmal transmutation \& transition among field genic units in space field; it's a numerical indication of dynamic intensity of connatural genic transmutation \& transition in space field.

The root cause time dynamic speed is but scalar quantity is that mutual phantasmal transmutation and transition of field genic units makes the existence of field genic units in spacetime absolutely inconstant, once any field genic unit performed any transmutation and transition, it immediately 
became "non-self", so that it's impossible for us to ascertain any definite reference to define orientation in space field, as a result, there is none definite orientation of genic transmutation and transition indeed. As a result, time dynamic speed can only be a scalar quantity indicating dynamic intensity or rapidness of phantasmal transmutation and transition of field genic units in space field. This of course indicates, as a very special speed reflecting intensity and rapidness of mutual transmutation and transition among field genic units in space field, time dynamic speed is substantively different from our conventional logos of speed.

2.2 Connotation objectification of logic extension 2 of Homogenous cosmos originated from unique genesis postulate,_Ultimate ascertainment of homologic filiation alternative between spacetime and matter leads to undeniable homologic generation of matter in homogenous cosmos

\subsubsection{Homologic generation of matter in homogenous cosmos}

In accordance with logic extension 2 of Homogenous cosmos originated from unique genesis postulate, if cosmos is object fully embodied by hypostatic coexistence of spacetime and matter, nothing else, along original cosmic demiurgic evolution of sequential homologic multiplication starting at the so-called common cosmic extremity genesis to cosmic status quo, "spacetime" and "matter" must be lineal homologic filiation.

Of course, It's easy for us to comprehend that homologic filiation between spacetime and matter apparently implies filiation alternative of "spacetime father, matter son" and "spacetime son, matter father". However, cosmic authenticity can only be coherently unique. What's the definite homologic filiation between spacetime and matter on earth? __ "pacetime father and matter son" or "matter father and spacetime son”?

Considering the solid authenticity of nature that matter exists in spacetime, we exclude the alternative possibility of homologic filiation of matter father and spacetime son. Because if the matter is father and spacetime is son really, before birth of the "son", where can the "father" exist and how to motion without spacetime? It's obviously incompatible against the actuality that matter exists and motions in spacetime. Whereas, if spacetime is father and matter is the son (matter is sequential homologic multiplication outgrowth of spacetime), it obviously seems compatible to the actuality that matter exists and motions in spacetime according to general behavioral logic sequence. Resultingly, we can affirm matter homologic generation as Matter homologic generation hypothesis as below according to alternative clarification of homologic filiation between spacetime and matter:

It's hypothesized that, along cosmic demiurgic evolution of sequential homologic multiplication, matter is successive outgrowth behiand hylic spacetime when spacetime develops to well-rounded stage, —_ some space field genic units congealed into "crystal" format for extra-saturation of space field.

This of course implies, on the basis of integrative precondition of "homogenous cosmos originated from unique genesis + spacetime-matter fully embodying cosmos", matter homologic generation hypothesis is further logic outcome of alternative clarification of homologic filiation between spacetime and matter by excluding alternative possibility of homologic filiation of matter father and spacetime son.

\subsection{Sum-up of connotation objectification of two logic extensions of Homogenous cosmos originated} from unique genesis postulate_logic outspread constitution of "Homogenous cosmos originated from unique genesis + spacetime matter fully embodying cosmos”.

As broken down representation of connotation objectification of logic extensions of "Homogenous cosmos originated from unique genesis + spacetime matter fully embodying cosmos, "space genic field hypothesis", "phantasmal genic unit hypothesis" and "matter homologic generation hypothesis" have to maintain their "hypothesis" identities. Above all, Homogenous cosmos originated from unique genesis postulate derived from newly highlighted factuality of any discretionary two particles in cosmos are mutually convertible, although the conclusion of the very factuality is apparently in living orientation towards perfect induction but still out of perfect induction at present, as a result, 
Homogenous cosmos originated from unique genesis postulate can only be introduced as a sensible postulate. And what's more, "space genic field hypothesis", "phantasmal genic unit hypothesis" and "matter homologic generation hypothesis" are but broken down representation of connotation objectification of Homogenous cosmos originated from unique genesis postulate with supplementation of spacetime matter fully embodying cosmos postulate, the broken down situation is rather similar as we can break down human body as "human body" = "head" + "the body" + "purtenance" + "extremities". Of course, we can also introduce similar equation to represent the broken down "connotation objectification of Homogenous cosmos originated from unique genesis postulate with supplementation of spacetime-matter fully embodying cosmos postulate" as below:

"Homogenous cosmos originated from unique genesis postulate" + "Spacetime-matter fully embodying cosmos postulate”

$\approx$ "Logic extension 1 of Homogenous cosmos originated from unique genesis postulate" + "Logic extension 2 of Homogenous cosmos originated from unique genesis postulate" ₹ ("space genic field hypothesis" + "Phantasmal field genic unit hypothesis") + "matter homologic generation hypothesis"

Something I have emphasize herein is that the precondition of logic extension 1 of Homogenous cosmos originated from unique genesis is "cosmos is homogenous originated from unique genesis + spacetime \& matter are all belonging to cosmos". However, I only mention "spacetime matter fully embodying cosmos" in above equation, but do not mention "spacetime \& matter are all belonging to cosmos", because, in logic connotation, "spacetime \& matter are all belonging to cosmos" covers the logic connotation of "spacetime \& matter are all belonging to cosmos" 100 over 100 . And just because of this, many occasions, I only mention "spacetime matter fully embodying cosmos" without "spacetime \& matter are coequally belonging to cosmos".

Upon strict sense, throughout radical objectification \& highlight of homologic attribute of spacetime \& matter, homologic filiation between spacetime \& matter and existence \& motion of matter in cosmos, Homogenous Cosmos Originated from Unique Genesis totally introduced “(1.0 + $\mathbf{0 . 5})+\mathbf{0 . 5}$ ” postulates. In another words, in order to illuminate cosmic homologic profile and consequential universal existence \& motion in cosmos, Homogenous Cosmos Originated from Unique Genesis introduced a couple cosmic mould cavities of Homogenous cosmos originated from unique genesis postulate, an accessorial slide of spacetime matter fully embodying cosmos postulate and a dynamic soul of Instinctive PNT equilibrium postulate.

The so-called " 1 " postulate is just the prime precondition of Homogenous cosmos originated from unique genesis postulate, which is just the couple cosmic mould cavities and the logic origin of the whole subject. In a straightforward word, all contents about homologic existence \& motion of matter in cosmos introduced in the monograph are but logic extension of the very postulate with supplementation of two " 0.5 ” grade postulates.

The first " 0.5 " grade auxiliary postulate is just the "Spacetime matter fully embodying cosmos postulate", it's a coordinate supplemental postulate like an accessorial slide of the couple mould cavities of Homogenous cosmos originated from unique genesis postulate, which hypothesizes spacetime and matter fully embody the entire cosmos, besides spacetime and matter, nothing else coordinative is existent in cosmos. The supplemental introduction of "Spacetime matter fully embodying cosmos postulate" directly make integrative precondition of "homogenous cosmos originated from unique genesis + spacetime matter fully embodying cosmos” logically extendable, and two logic extensions of Homogenous cosmos originated from unique genesis postulate are deduced thereby.

The second " $\mathbf{0 . 5}$ " grade auxiliary postulate is "Instinctive PNT equilibrium postulate" cosmic dynamic soul. The reason I call it the second " $\mathbf{0 . 5}$ ” grade auxiliary postulate is but because it's a supplemental postulate for consummate linkage \& transition from logic extension and connotation objectification of "homogenous cosmos originated from unique genesis + spacetime matter fully embodying cosmos" to definite "existence \& motion of matter" in cosmos.

Once logic extension and proper connotation objectification of "homogenous cosmos originated from unique genesis + spacetime matter fully embodying cosmos” approaches alternative ascertainment of 
homologic filiation between spacetime and matter, as long as we exclude possibility of matter father and spacetime son, homologic generation of matter in cosmos is self-evident. And homologic filiation between spacetime and matter directly indicates presence of PNT action, PNT action is not only headspring action bringing up original homologic generation of matter but also dynamic origin of consequential existence \& motion of matter in spacetime, which apparently indicates existence \& motion of matter undeniably root in PNT action of matter integration.

However, what magic action is "PNT action" on earth?

At any rate, it's absolutely impossible for PNT action to be blackguardly action of the God. Since PNT action radically determines existence \& motion of matter, there must be some definite dynamic fundamental of PNT action underlying which indicates sense of the God, otherwise, there is no way for cosmos to be so well-regulated. Whereas, to my despair, it's almost impossible for me to ascertain the definite dynamic fundamental of PNT action underlying on the spot, but to suppose it may be generally "instinctive PNT equilibrium" according to remarkable experiential factuality around after confusion for a long time.

Virtually, only the supplemental introduction of "Instinctive PNT equilibrium postulate" can consummate the rational transition from logic extension and proper connotation objectification of "homogenous cosmos originated from unique genesis + spacetime matter fully embodying cosmos" to definite "existence \& motion of matter" in cosmos, —-spontaneous rebuilding of PNT action of matter integration due to instinctive PNT equilibrium towards optimum PNT operation.

The integrative logic constitution of logic extension in logic model of “(1.0 $+\mathbf{0 . 5})+\mathbf{0 . 5}$ ” is generally as below:

(Homogenous cosmos originated from unique genesis postulate + spacetime matter fully embodying cosmos postulate) + Instinctive PNT equilibrium postulate

$\approx$ (Logic extension 1 of Homogenous cosmos originated from unique genesis postulate + Logic extension 2 of Homogenous cosmos originated from unique genesis postulate) + Instinctive PNT equilibrium postulate

z[(spacetime genic field hypothesis + Phantasmal field genic unit hypothesis ) + Matter homologic generation hypothesis] + Instinctive PNT equilibrium postulate

$\approx$ Spontaneous occurrence of "PNT action + Instinctive PNT equilibrium" of matter integration under integrative background of homogenous cosmos originated from unique genesis

= Vivid existence \& motion of matter in cosmos under integrative background of homogenous cosmos originated from unique genesis

\section{Acknowledgement}

This paper is excerpted from the first part and the second part of my monograph of Homogenous Cosmos Originated from Unique Genesis (http://www.docin.com/p1-2010790280.html). And before my submission, Dr huangjun attended language editting of the paper.

\section{References}

[1] Yang guosheng, Historical progress of science, Wuhan, hans publication organization 2013

[2] Myatt C J, King B E, Wineland D J, et al. Decoherence of quantum superpositions through coupling to engineered reservoirs. Nature, 2000,403: 269-273

[3] Marshall T W. Brownian motion of a mirror. Phys. Rev. D, 1981, 24(6): 1509 1515. 